\title{
Social Media in the Medical Laboratory Workplace: A Literature Review
}

HARRY MCDONALD JR, CINDY HANDLEY

\begin{abstract}
Social media has become a source of debate in the medical laboratory community as to what is appropriate and what is inappropriate for sharing through online communication. As of today, more and more medical institutions are reaching outside their secure network in soliciting social media forums, like Twitter and Facebook, to actively engage and directly communicate with their constituents, patients or staff. In addition, institutions are generating original content and encouraging discussion about their techniques and services through blogs and chat rooms on their websites. The ever-expanding world of social media has become a contentious subject for many institutions, as their employees' usage may blur the lines between what is professional and personal in the workplace. Although it still applies, social media creates the effect that it removes boundaries regarding privacy and confidentially as seen in the workforce setting. Despite the relatively widespread adoption of social media among medical institutions, many lack internal policies or systems to adequately address protocol of when, how and where to use social media. Reciprocally, as the number of smartphones, tablets and personal laptops increase in usage, so will the use of social media in the medical laboratory workplace. Undoubtedly, as this increase occurs, the establishment of policies and procedures will be essential in addressing proper protocol regarding social media usage in the workplace.
\end{abstract}

INDEX TERMS: social media, communication, confidentiality, privacy, medical workplace

\section{Clin Lab Sci 2015;28(4):209-211}

Harry McDonald Jr., MS, MT(ASCP), SBB, University of Southern Mississippi, Hattiesburg, MS

Cindy Handley, PhD, MT(ASCP), University of Southern Mississippi, Department of Medical Laboratory Science, Hattiesburg, MS
Address for Correspondence: Harry McDonald Jr., MS, MT(ASCP), SBB, University of Southern Mississippi, 118 College Drive, Box 5134, Hattiesburg, MS,301-792-7542, harry.mcdonaldjr@us.army.mil

\section{INTRODUCTION}

This literature review focuses on the effect of social media in the medical laboratory workplace. Social media includes electronic information created or posted externally online, for example, but are not limited to: text messages, media messaging services, Twitter, Facebook, Linked-In, Pinterest, YouTube, Instagram and all other social networks. ${ }^{1}$

Social media has been in existence for over 40 years and, as time progresses, digital natives (age group 15-24, individuals who have grown up in a digital world) will only increase their prominence in medical institutions. In the medical workplace, social media can be a valuable tool if used appropriately. It can facilitate the access to collective intelligence, creativity and provide invaluable information in improving healthcare initiatives. ${ }^{2}$

\section{Credibility}

However, if misused, serious concerns may occur jeopardizing the credibility of the individual and the entire medical institution. Some examples of inappropriate use of social media in the medical work place are as follows. In 2008, a group of nurses used Facebook to approve unauthorized shift change reports containing patient information; this information was disclosed to friends and relatives which may be a violation of federal privacy and regulations acts. ${ }^{3}$ Another inappropriate usage of social media was from an employee who took pictures of a patient's buttocks at a hospital emergency room, and posted the pictures to friends (colleagues) on social media in which they made inappropriate comments. Although the patient's face was not shown, the hospital Human Resource Department deemed this inappropriate and terminated all employees who 'liked' the picture along with the 


\section{CLINICAL PRACTICE}

original poster. ${ }^{4}$ These are examples that demonstrate the ease at which professional and personal relations are quickly blurred or confused for employees who use social media in the workplace. The inundation of new technology by digital natives will require medical institutions to develop social media policies that embody the positive benefits of social media use, while minimizing the negative features.

\section{Legal Aspects}

The ultimate goal of social media for the medical laboratory science community is to present accurate and precise information to constituents that permits transparency, easy access, and speed for medical beneficial purposes. $^{5}$ Conversely, in sharing this information institutions must conform to the Health Information Portability and Accountability Act of 1996 (HIPPA), which limits the use of patient data to only those who are authorized to see it in special cases. ${ }^{6}$ Social media often gives the users a false sense of security, with the assumption that audiences are well defined within a specific realm on a particular topic. Social media has its drawbacks, in the fact that information shared online is no longer under the control of the author, allowing other individuals to forward information on to other users without the original author's consent. In addition, the release of information on social media leaves a digital footprint that is traceable back to the author. Other drawbacks such as site security settings may not offer true exclusion of the public. For example, certain sites share user information without the user consent, and group users of the social site may compromise privacy by the inattentiveness of their security settings. ${ }^{2}$

Social media are new tools to improve instructional education in the classroom and laboratory settings. While free speech cannot be constrained, this new approach to education comes with great responsibility for the institution to control the access and behavior of students on social media. According to Rice, "Federal court rulings on social media posts of nursing students posing with placentas, medical students posing with cadavers and mortuary students' thoughts on anatomy labs, raise concerns about how professionalism, privacysecurity, intellectual property and mobile devices are addressed in educational policies". ${ }^{6}$

Students without appropriate training may breach
HIPPA policy, by inadvertently sharing downloads across multiple social network platforms or using mobile devices that place private information on unsecure server clouds. Rice further states that, "Court rulings place responsibility on educational institutions to teach professional behavior and conduct of students' use of social media". ${ }^{6}$ These rulings clearly explain that students are not held accountable for posts on social media if training policies are not in place; therefore, it is essential that institutions have policies in place for the use of social media.

\section{Formal and informal surveys}

An online survey was conducted by Cunningham (2014) that investigated the perception of the medical students, teachers, physicians and patients at the University of Michigan toward social media using mock medical students' Facebook profiles. The mock profiles consisted of derogatory and/or private information about patient information. For each profile the groups rated the appropriateness (would they be comfortable with having the student profile as their future doctor) of the profile on a Likert-like scale of one to five (with five being the most appropriate). The results of the study demonstrated that all groups rated the mock profiles as inappropriate. This study provides veracity showing that there is a need for social media policy and inservice training to ensure students consider the perspectives of patients before posting content on social media sites. ${ }^{7,8}$

In the medical workplace, social networking continues to be a huge subject regarding social behavior because the separation of professional and private lives seems to have no delineation. While it is possible to check personal information during professional time, one must pay attention to institutions' policies (if one exists) when posting to social networks. Mistakes that affect work performance can easily occur if an individual is using a mobile device to conduct professional and personal business simultaneously. An accident occurred in one such case where a medical resident used a smartphone for both personal and professional business. As he was entering medical orders during ward rounds, the individual was interrupted by a personal text invitation to a party. The resident became distracted; the order regarding the patient was never completed, resulting in an adverse patient event. ${ }^{2}$ 
Although there is not much literature regarding social media in the medical laboratory work place, an informal survey of the local hospitals and clinics was revealing regarding social media policies in the workplace. The results of the survey revealed that 10 out of 10 surrounding hospitals and clinics visited did not have internal clinical laboratory policies to address the proper use of social media. Collectively, when the staff was questioned at all locations about policies and procedures of social media, they replied that employees were not allowed to use social media at work. Nevertheless, all members that were questioned had smartphones and implied they used social media at work during work hours and personal time. Most medical institutions believe that a general blanket statement will control the use of social networking during work hours. However, after reviewing articles and questioning administrators in local hospitals and clinics, it was determined that a general practice of implementing internal policies and in-services are critical to circumvent the problems that are on the horizon due to the surge of social media in medical institutions.

\section{CONCLUSION}

The medical laboratory community appears to lack policies to control proper use of social media in the work environment. Most medical institutions prohibit the use of social media during work hours on institution owned computers, but this prohibition often does not extend to the use of personal smartphones and tablets, which are accessible to most individuals. Social media can be useful in health care settings but must be monitored for misuse on a regular basis to keep in compliance with HIPPA and to remain ethically stable. ${ }^{5}$

Social media has greatly changed our professional and personal lives. These changes bring a new element to the clinical laboratory profession, offering a platform used by health professionals to communicate health and health issues expeditiously and in a user friendly way. ${ }^{5}$
However, with this technology, institutions must stay abreast of the changes of technologies and regulations. Conversely, if left unsupervised, social media has the potential to leave a trail of inexcusable damage that may affect institutions' creditability and future business practices. ${ }^{9}$

\section{ACKNOWLEDGEMENTS}

The authors would like to acknowledge Mrs. Shannon Goolsby for bringing this topic to our attention and performing some original research on the educational aspect of the use of social media in the workplace.

\section{REFERENCES}

1. Santoro E. Internet and social media for health-related information and communication in health care. Recenti Prog Med, 2013 Mar 11;104 (5),179-80. doi 10.1701/1291.14273.

2. Ayres EJ. The impact of social media on business and ethical practices in dietetics. J Acad Nutr Diet, 2013 Nov 1; 113(11): 1539-43. doi.org/10.1016.

3. Davis J. Nurses fired for online social media posts. Nursing show [Internet]. 2013 [cited 2013 Apr 19]. Available from: http://www.nursingshow.com/2013/page/5/

4. Spectrum workers fired over Facebook picture. WZZM 13 Television [Internet]. 2013 [cited 2013 April]. Available from: www.wzzm 13.com/news/article/266439/2/spectrum-health employee-fired-over-facebook-picture/

5. Moorhead SA, Hazlett DE, Harrison L, Carroll JK, Irwin A., Hoving C. A new dimension of health care: systematic review of the uses, benefits, and limitations of social media for health communication. Journal of Medical Internet Research, 2013 23 Apr 23;15(4),85. Retrieved on 21 February 2014.

6. Rice MJ. Social Media in Healthcare: Educational Policy Implications. Arch Psychiatr Nurs, 2013 Feb 1; 27(1): 61-62 doi:10.1016/j.apnu.2012.11.001.

7. Cunningham A. Social media and medical professionalism. Med Educ, 2014 48(2):110-2. doi: 10.1111/medu.12404.

8. Jain A, Petty EM, Jaber RM, Tackett S, Purkiss J, Fitzgerald J, White, J. What is appropriate to post on social media? Ratings from students, faculty members and the public. Med Educ 2014 Feb; 48(2):157-69. doi: 10.1111/medu.12282.

9. Grajales FJ, Sheps S, Ho K., Novak-Lauscher H, \& Eysenbach G. Social Media: A Review and Tutorial of Applications in Medicine and Health Care. J Med Internet Res, 2014 Feb 11;16(2):13. doi:10.2196/jmir.2912. 